\title{
The Eccentric Kozai-Lidov Mechanism for Outer Test Particle
}

\author{
Smadar Naoz ${ }^{1,5}$, Gongjie $\mathrm{Li}^{2}$, Macarena Zanardi ${ }^{3,4}$, Gonzalo Carlos de Elía, ${ }^{3,4}$, and Romina P. Di Sisto ${ }^{3,4}$ \\ ${ }^{1}$ Department of Physics and Astronomy, University of California, Los Angeles, CA 90095, USA; snaoz@astro.ucla.edu \\ ${ }^{2}$ Harvard Smithsonian Center for Astrophysics, Institute for Theory and Computation, 60 Garden Street, Cambridge, MA 02138, USA \\ 3 Instituto de Astrofísica de La Plata, CCT La Plata-CONICET-UNLP Paseo del Bosque S/N (1900), La Plata, Argentina \\ ${ }^{4}$ Facultad de Ciencias Astronómicas y Geofísicas, Universidad Nacional de La Plata Paseo del Bosque S/N (1900), La Plata, Argentina \\ ${ }^{5}$ Mani L. Bhaumik Institute for Theoretical Physics, Department of Physics and Astronomy, UCLA, Los Angeles, CA 90095, USA \\ Received 2017 January 9; revised 2017 April 20; accepted 2017 April 24; published 2017 June 21
}

\begin{abstract}
The secular approximation of the hierarchical three body systems has been proven to be very useful in addressing many astrophysical systems, from planets to stars to black holes. In such a system, two objects are on a tight orbit and the tertiary is on a much wider orbit. Here, we study the dynamics of a system by taking the tertiary mass to zero and solve the hierarchical three body system up to the octupole level of approximation. We find a rich dynamics that the outer orbit undergoes due to gravitational perturbations from the inner binary. The nominal result of the precession of the nodes is mostly limited for the lowest order of approximation; however, when the octupole level of approximation is introduced, the system becomes chaotic, as expected, and the tertiary oscillates below and above $90^{\circ}$, similarly to the non-test particle flip behavior. We provide the Hamiltonian of the system and investigate the dynamics of the system from the quadrupole to the octupole level of approximations. We also analyze the chaotic and quasi-periodic orbital evolution by studying the surfaces of sections. Furthermore, including general relativity, we showcase the long-term evolution of individual debris disk particles under the influence of a far-away interior eccentric planet. We show that this dynamics can naturally result in retrograde objects and a puffy disk after a long timescale evolution (a few Gyr) for initially aligned configuration.
\end{abstract}

Key words: celestial mechanics - chaos - minor planets, asteroids: general - planetary systems

\section{Introduction}

The hierarchical three body secular dynamics has been studied extensively in the literature and was shown to be very effective in addressing different astrophysical phenomena (see, for review, Naoz 2016, and reference therein). In this hierarchical setting, the inner binary is orbited by a third body on a much wider orbit, the outer binary, such that the secular approximation can be applied (i.e., phase averaged, long-term interaction). The gravitational potential is then expanded in semimajor axis ratio $\left(a_{1} / a_{2}\right.$, which, in this approximation, remains constant), where $a_{1}\left(a_{2}\right)$ is the semimajor axis of the inner (outer) body (Kozai 1962; Lidov 1962). This ratio is a small parameter due to the hierarchical configuration. The lowest order of approximation, which is proportional to $\left(a_{1} / a_{2}\right)^{2}$ is called the quadrupole-level.

Most of these studies focus on the gravitational perturbations that a far-away perturber exerts on the inner binary. In early studies of high-inclination secular perturbations (Kozai 1962; Lidov 1962), the outer orbit was assumed to be circular, and it was assumed that one of the inner binary members is a massless test particle. In this situation, the component of the inner orbit's angular momentum along the $z$-axis (which is set to be parallel to the total angular momentum, i.e., the invariable plane) is conserved, and the lowest order of the approximation, the quadrupole approximation, is valid. However, relaxing either one of these assumptions leads to qualitative different behavior (e.g., Katz et al. 2011; Lithwick \& Naoz 2011; Naoz et al. 2011). Considering systems beyond the test particle approximation, or a circular orbit, requires the next level of approximation, called the octupole level of approximation, which is proportional to $\left(a_{1} / a_{2}\right)^{3}$ (e.g., Harrington 1968, 1969; Ford et al. 2000; Blaes et al. 2002). In the octupole level of approximation, the inner orbit eccentricity may reach extreme values (Ford et al. 2000; Naoz et al. 2013a; Teyssandier et al. 2013; Li et al. 2014c). In addition, the inner orbit can flip its orientation, with respect to the total angular momentum (i.e., $z$-axis), from prograde to retrograde (Naoz et al. 2011).

Here, we study the secular evolution of a far-away test particle orbiting an inner massive binary. In this case, the inner orbit is fixed, and effectively carries all of the annular momentum of the system, while the outer orbit undergoes a dynamical evolution. This situation has a large range of applications from the gravitational perturbations of binary supermassive black holes on the surrounding stellar distribution to the effects of planetary orbits on debris disks, Oort cloud, and trans-Neptunian objects. From $\mathrm{N}$-body simulations, Zanardi et al. (2017) analyzed the long-term evolution of test particles in the presence of an interior eccentric planet. Such a study produces particles on prograde and retrograde orbits, as well as particles whose orbital plane flips from prograde to retrograde and back again along their evolution.

We note that Ziglin (1975) investigated the oscillations of an outer circumbinary planet in the context of the restricted elliptical three body problem. Later Verrier \& Evans (2009) and Farago \& Laskar (2010) studied the stability of a high inclined planet around in this situation using a combination of numerical and perturbation theory up to the quadruple level of approximation approaches (see also, Li et al. 2014a; de la Fuente Marcos et al. 2015). Furthermore, Gallardo (2006) and Gallardo et al. (2012) studied the effects of the Kozai-Lidov for trans-Neptunian objects near mean motion resonance with Neptune. However, here, we do not allow for mean motion resonances to allow for the double averaging process (see $\mathrm{NaOz}$ et al. 2013a, Appendix A2 for the canonical transformation, which describes the averaging process). We provide a general 
treatment for the outer test particle case, up to the octupole level of approximation in the secular theory.

The paper is organized as follow: we begin by describing the outer test particle Hamiltonian and equations of motion (Section 2) and continue to discuss the quadrupole-level of approximation (Section 3), where we also drive the relevant timescales. Then, we study the role of the octupole level of approximations and provide surface of section maps in Section 4. We also discuss the role of general relativity (GR) precession in Section 5. We then consider one case study in the form of the long-term evolution debris disk particles in Section 6. Finally, we offer our discussions in Section 7.

\section{The Equations of Motion}

We solve the orbit of an exterior massless test particle to an eccentric planet $\left(m_{2}\right)$, both orbiting a star $\left(m_{1}\right)$, including only secular interactions expanded to octupole order. The planet is on a fixed eccentric orbit (i.e., $e_{1}=$ const) and the outer particle's orbit is specified by four variables:

$$
e_{2}, \omega_{2}, \theta, \Omega_{2}
$$

where $e_{2}$ is the test particle eccentricity, $\theta=\cos i$ and $i$ is the inclination of the test particle with respect to the inner orbit, and $\omega_{2}$ and $\Omega_{2}$ are the argument of periapse and longitude of ascending node of the outer orbit, relative to the inner planet's periapse, respectively (Murray \& Dermott 2000). Specifically, we set $\varpi_{1}=0$ (see Appendix A for the coordinate transformation). We kept the subscript " 2 " in $\omega_{2}$ and $\Omega_{2}$ for consistency with the comparable masses treatments. From $e_{2}$ and $\theta$ we can define the canonical specific momenta

$$
\begin{gathered}
J_{2}=\sqrt{1-e_{2}^{2}} \\
J_{2, z}=\theta \sqrt{1-e_{2}^{2}} .
\end{gathered}
$$

The Hamiltonian for which $m_{3} \rightarrow 0$ is

$$
f=f_{\text {quad }}+\epsilon_{M} f_{\text {oct }} \text {, }
$$

where

$$
\epsilon_{M}=\frac{m_{1}-m_{2}}{m_{1}+m_{2}} \frac{a_{1}}{a_{2}} \frac{e_{2}}{1-e_{2}^{2}}
$$

and

$$
\begin{aligned}
f_{\text {quad }}= & \frac{\left(2+3 e_{1}^{2}\right)\left(3 \theta^{2}-1\right)+15 e_{1}^{2}\left(1-\theta^{2}\right) \cos \left(2 \Omega_{2}\right)}{\left(1-e_{2}^{2}\right)^{3 / 2}}, \\
f_{\text {oct }}= & \frac{15 e_{1}}{4\left(1-e_{2}^{2}\right)^{3 / 2}}\left[10\left(1-e_{1}^{2}\right) \theta\left(1-\theta^{2}\right) \sin \omega_{2} \sin \Omega_{2}\right. \\
& +\frac{1}{2}\left\{2+19 e_{1}^{2}-5\left(2+5 e_{1}^{2}\right) \theta^{2}\right. \\
& \left.-35 e_{1}^{2}\left(1-\theta^{2}\right) \cos \left(2 \Omega_{2}\right)\right\} \\
& \left.\times\left(\theta \sin \omega_{2} \sin \Omega_{2}-\cos \omega_{2} \cos \Omega_{2}\right)\right] .
\end{aligned}
$$

Note that unlike the inner test particle approximation, $\epsilon_{M}$ is not constant during the motion and the constant parameter during the evolution is

$$
\delta=\frac{m_{1}-m_{2}}{m_{1}+m_{2}} \frac{a_{1}}{a_{2}} e_{1}
$$

and the Hamiltonian up to the octupole level of approximation can be defined as

$$
\tilde{f}_{\mathrm{oct}}=\frac{e_{2}}{1-e_{2}^{2}} \frac{f_{\mathrm{oct}}}{e_{1}}
$$

and

$$
f=f_{\text {quad }}+\delta \tilde{f}_{\text {oct }} .
$$

We note that $f_{\text {quad }}$ has the same functional form as the inner test particle $F_{\text {quad }}$ presented in Lithwick \& Naoz (2011) up to the $\left(1-e_{2}^{2}\right)^{3 / 2}$, which is not constant in our case.

The equations of motion may be expressed as partial derivatives of an energy function $f\left(e_{2}, \omega_{2}, \theta, \Omega_{2}\right)$

$$
\begin{gathered}
\frac{d J_{2}}{d \tau}=\frac{\partial f}{\partial \omega_{2}} \\
\frac{d J_{2, z}}{d \tau}=\frac{\partial f}{\partial \Omega_{2}} \\
\frac{d \omega_{2}}{d \tau}=\frac{\partial f}{\partial e_{2}} \frac{J_{2}}{e_{2}}+\frac{\partial f}{\partial \theta} \frac{\theta}{J_{2}} \\
\frac{d \Omega_{2}}{d \tau}=-\frac{\partial f}{\partial \theta} \frac{1}{J_{2}}
\end{gathered}
$$

where $\tau$ is proportional to the true time (see Equation (29)). Unlike the inner orbit test particle, $\epsilon_{M}$ is not constant while $e_{1}$ is constant (in other words, the angular momentum of the inner orbit is conserved). The equations of motion were tested successfully compared to the general equations of motions, presented in Naoz et al. (2013a). We also test the evolution compared to $\mathrm{N}$-body in Appendix B.

\section{Quadrupole-level of Approximation}

\subsection{General Analysis}

The quadrupole-level of approximation is integrable and thus provides a good starting point. Unlike the quadrupole-level approximation for the inner orbit test particle, here, the $z$ component of the particle's angular momentum is not conserved, as the Hamiltonian depends on $\Omega_{2}$. However, at this level $J_{2}$ is conserved, and thus the outer orbit eccentricity $e_{2}$ remains constant as the Hamiltonian does not depends on $\omega_{2}$. The equation of motion for the inclination takes a simple form

$$
\begin{aligned}
\left.\frac{d \theta}{d t}\right|_{\text {quad }}= & -\frac{15}{8}\left(\frac{a_{1}}{a_{2}}\right)^{2} \frac{2 \pi}{P_{2}} \frac{m_{1} m_{2}}{\left(m_{1}+m_{2}\right)^{2}} e_{1}^{2} \\
& \times \frac{1-\theta^{2}}{\left(1-e_{2}^{2}\right)^{2}} \sin 2 \Omega_{2},
\end{aligned}
$$

where we consider the time evolution and not the scaled evolution for completeness, and $P_{2}$ is the period of the outer orbit. We can find the maximum and minimum inclination by setting $\dot{\theta}=0$. Thus, we find that the values of the longitude of ascending nodes that satisfy this condition are $\Omega_{2}=n \pi / 2$, where $n=0,1,2 \ldots$ In other words, $\Omega_{2}$ has two classes of trajectories, librating and circulating. The trajectories in the librating region are bound between two values of $\Omega_{2}$, while the circulation region represents trajectories where the angles are not constrained between two specific values. On circulating trajectories, at $\Omega_{2}=0$, the inclination $\left(i<90^{\circ}\right)$ is largest 


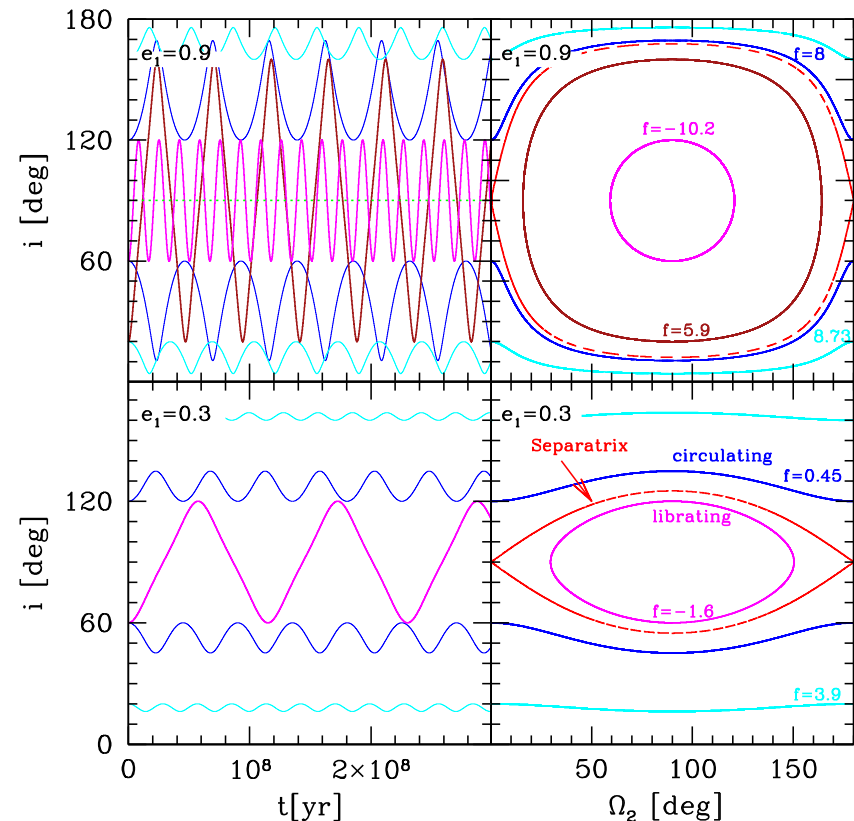

Figure 1. The quadrupole-level of approximation evolution. We show the time evolution of the inclination in the left panels and the cross-section trajectory in the inclination $-\Omega_{2}$ plane, in the right panels. We consider two cases, the top panels are for $e_{1}=0.9$ and the bottom panels are for $e_{1}=0.3$. We show the following examples: in the circulating mode (setting initially $\Omega_{2}=0$ ): $i=20^{\circ}$ (cyan), $60^{\circ}$ (blue), and in the librating mode (setting initially $\Omega_{2}=90^{\circ}$ ): $i=20^{\circ}$ (brown) and $60^{\circ}$ (magenta). The separatrix corresponds to $i=90^{\circ}$ for the two different inner eccentricities is shown in red. Note that in the case of $e_{1}=0.3$ there is no librating mode for $i=20^{\circ}$. For consistency we adopt the following orbital parameters: $m_{1}=1 M_{\odot}, m_{1}=1 M_{J}, a_{1}=3$ au and $a_{2}=$ $40 \mathrm{au}, \omega_{2}=90^{\circ}$ and $e_{2}=0$.

(where the $i>90^{\circ}$ case is a mirror image of the $i<90^{\circ}$ one). The extrema points for the librating mode are located at $\Omega_{2}=90^{\circ}$. The time evolution of $\Omega_{2}$ for the quadrupole-level of approximation is given by

$$
\begin{aligned}
\left.\frac{d \Omega_{2}}{d t}\right|_{\text {quad }}= & -\frac{m_{1} m_{2}}{\left(m_{1}+m_{2}\right)^{2}} \frac{2 \pi}{P_{2}}\left(\frac{a_{1}}{a_{2}}\right)^{2} \\
& \times \frac{3 \theta\left(2+3 e_{1}^{2}-5 e_{1}^{2} \cos 2 \Omega_{2}\right)}{8\left(1-e_{2}^{2}\right)^{2}} .
\end{aligned}
$$

In Figure 1, we show the evolution associated for the quadrupole-level of approximation. The two librating and circulating trajectories are considered, where we folded the $\Omega_{2}$ angle to be between $0^{\circ}-180^{\circ}$. The librating mode gives the nominal precession of the nodes, at which the inclination oscillates between the $i_{90}$ inclination (the inclination for which $\Omega_{2}=90^{\circ}$ ) and $180^{\circ}-i_{90}$. The precession of the nodes was noted before in the literature (e.g., Innanen et al. 1997).

From the latter equation and Equation (15) we have

$$
\frac{d \Omega_{2}}{d \theta}=\theta \frac{2+3 e_{1}^{2}-5 e_{1}^{2} \cos 2 \Omega_{2}}{5 e_{1}^{2}\left(1-\theta^{2}\right) \sin 2 \Omega_{2}} .
$$

Integrating the two sides we have

$$
\int_{\Omega_{b}}^{\Omega_{a}} \frac{\sin 2 \Omega_{2}}{2+3 e_{1}^{2}-5 e_{1}^{2} \cos 2 \Omega_{2}} d \Omega_{2}=\int_{\theta_{a}}^{\theta_{b}} \frac{\theta}{5 e_{1}^{2}\left(1-\theta^{2}\right)} d \theta,
$$

where $\Omega_{a, b}$ is the longitude of ascending nodes that is associated with the inclination value of $\theta_{a, b}=\cos i_{a, b}$.

For the circulating mode, we find that, after integrating over Equation (17) from $i_{\max }$ to $i_{\min }$ (and $\Omega_{2}=0$ to $\Omega_{2}=90^{\circ}$, respectively) we get

$$
\sin i_{\min }=\sin i_{\max } \sqrt{\frac{1-e_{1}^{2}}{1+4 e_{1}^{2}}} .
$$

Note that this expression can be also achieved by considering the conservation of energy between the minimum and maximum cases. Setting the initial conditions for the energy, Equation (6), we can find the extrema points as a function of the initial conditions. A special case can be considered when $\Omega_{2}$ is initially set to be zero, and then the maximum inclination is the initial inclination $i_{0}$, so that

$$
\sin i_{\min }=\sin i_{0} \sqrt{\frac{\left(1-e_{1}^{2}\right)}{\left(1+4 e_{1}^{2}\right)}} \text { for } \quad \Omega_{2,0}=0^{\circ} .
$$

In other words, we can set $i_{\max } \rightarrow 90^{\circ}$ and, for a given $e_{1}$, find the largest $i_{\min }$ allowed, which corresponds to the separatrix. This relationship is also apparent in the numerical results of Figure 14 in Zanardi et al. (2017). As can be seen, for the circulating mode depicted in Figure 1 , setting initially $i=20^{\circ}$ corresponds to $i_{\min }=16^{\circ} .25$ and 10.56 for $e_{1}=0.3$ and $e_{1}=0.9$, respectively, consistent with Equation (20). A comparison between the flip criterion and the numerical results can be seen in Figure 9.

For the librating mode, we set $i_{\min }$, which is associated with the $\Omega_{2}=90^{\circ}$ case, and thus integrating over Equation (17) between $i=90^{\circ}$ to $i_{\min }$ (and $\Omega_{2}=\Omega_{2, \min }$ to $\Omega_{2}=90^{\circ}$, respectively), we get,

$$
\cos 2 \Omega_{2, \min }=\frac{2+3 e_{1}^{2}-\left(2+8 e_{1}^{2}\right) \sin ^{2} i_{\min }}{5 e_{1}^{2}} .
$$

Therefore, setting initially $\Omega_{2}=90^{\circ}$ as in the examples depicted in Figure 1, we find that the minimum value that $\Omega_{2}$ can achieve in the $e_{1}=0.9$ case is $59^{\circ} .23$ for the $i_{\min }=60^{\circ}$ and $15^{\circ} .95$ for the $i_{\min }=20^{\circ}$ example, consistent with the numerical results. Hence, the range of which $\Omega_{2}$ is librating on is $2 \times\left(90^{\circ}-\Omega_{2, \min }\right)$.

\subsection{Timescale}

The timescale associated with the evolution can be estimated from the equation of motion for $\Omega_{2}$ at the quadrupole-level (Equation (16)), for the circulating mode, by setting $d \Omega_{2} \rightarrow \pi$ and taking the terms in the parenthesis to be roughly order of unity (which is achieved, by setting $\Omega_{2} \rightarrow 0$ ):

$$
t_{\text {quad }} \sim \frac{4}{3} P_{2}\left(1-e_{2}^{2}\right)^{2} \frac{\left(m_{1}+m_{2}\right)^{2}}{m_{1} m_{2}}\left(\frac{a_{2}}{a_{1}}\right)^{2} \quad \text { circulating. }
$$

For the example system depicted in Figure 1, this equation gives a timescale of about $6 \times 10^{7}$ year, for an initial inclination of $60^{\circ}$, which agrees with the circulating mode (although we note that different $e_{1}$ give slightly different timescales). We also estimate the timescale in the librating mode by setting $d \Omega_{2} \rightarrow 2 \times\left(90^{\circ}-\Omega_{2 \text {, min }}\right)$, which we have 

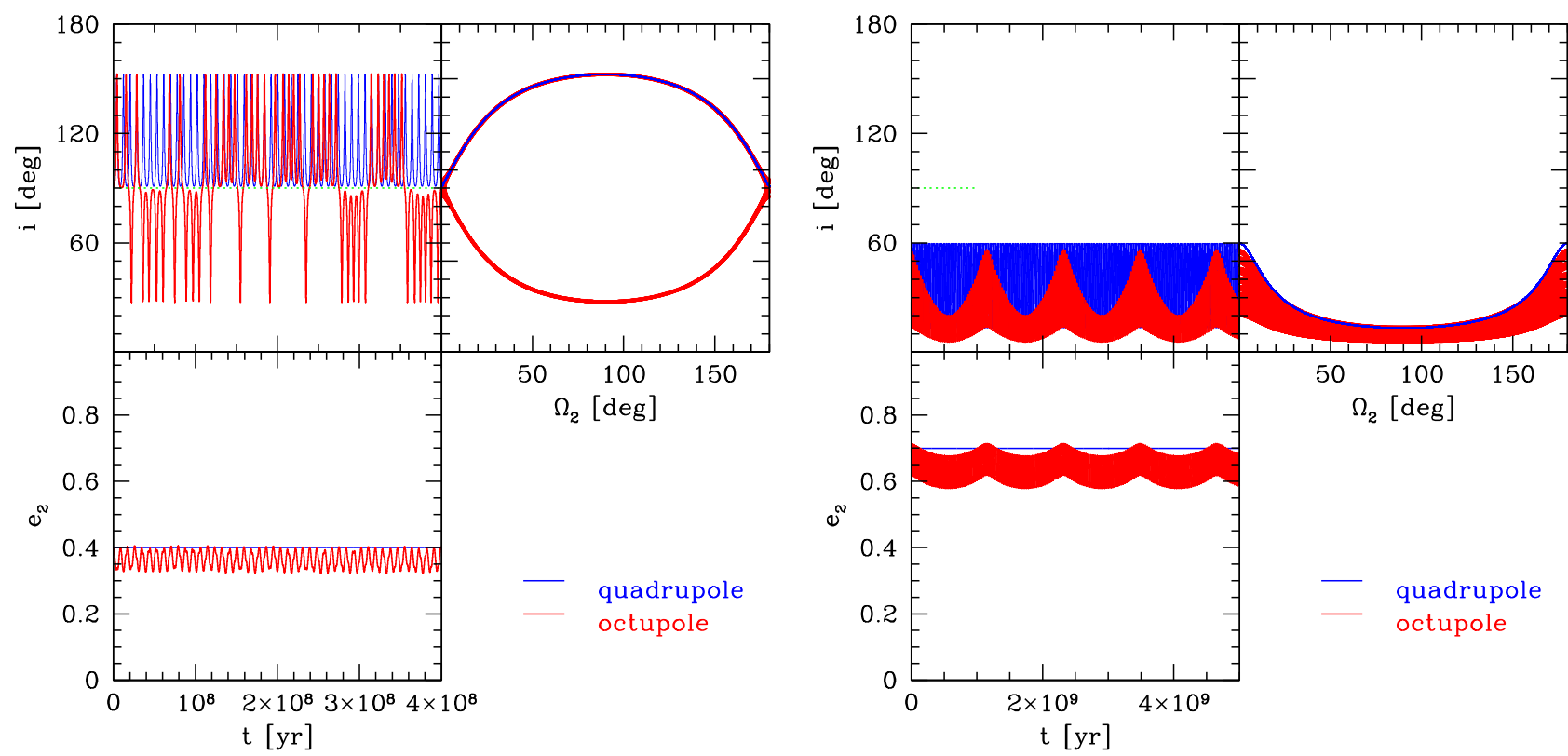

Figure 2. The role of octupole. We consider two cases, the secular evolution up to the quadrupole-level of approximation (blue lines) and up to the octupole level of approximation (red lines). We show the time evolution of the inclination and the outer orbital eccentricity (which remains constant at the quadrupole-level of approximation). We also consider the inclination evolution as a function of $\Omega_{2}$. Left panels: we consider the following system: $m_{1}=1 M_{\odot}, m_{2}=1 M_{j}, a_{1}=0.4$ au, $a_{2}=7 \mathrm{au}, e_{1}=0.65$ and $e_{2}=0.4$. We initialize the system with $\omega_{2}=\Omega_{2}=0^{\circ}$ and $i=91^{\circ}$. Right panels: $m_{1}=1 M_{\odot}, m_{2}=1 M_{j}, a_{1}=3$ au, $a_{2}=50$ au, $e_{1}=0.85$ and $e_{2}=0.7$. We initialize the system with $\omega_{2}=0^{\circ}, \Omega_{2}=40^{\circ}$ and $i=20^{\circ}$.

found earlier. Thus,

$$
\begin{aligned}
t_{\text {quad }} \sim & 2 \times \frac{2\left(\pi / 2-\Omega_{2, \min }\right)}{2 \pi} \frac{8}{3} P_{2}\left(1-e_{2}^{2}\right)^{2} \\
& \times \frac{\left(m_{1}+m_{2}\right)^{2}}{m_{1} m_{2}}\left(\frac{a_{2}}{a_{1}}\right)^{2} \quad \text { librating. }
\end{aligned}
$$

The pre-factor of two here comes from numerical comparisons to the examples depicted in Figure 1. Note the $e_{1}$ dependency that rises from $\Omega_{2, \min }$. This timescale is consistent with the examples depicted in Figure 1 by less than a factor of two.

\section{The Role of the Octupole Level of Approximation}

The octupole level of approximation can significantly affect the overall dynamics of the general hierarchical three body system (see Naoz 2016, and reference therein). Specifically, in the inner test particle case, the inner orbit's z-component of the angular momentum is not conserved anymore, and the orbit is allowed to flip (for a large range of initial inclinations Lithwick \& Naoz 2011; Li et al. 2014b, 2014c). In our case, the $J_{2, z}$ is not conserved at the quadrupole-level, but $J_{2}$ is. Thus, the octupole level of approximation in this case allows for variations of $e_{2}$ and introduces higher-level resonances, which may result in a chaotic behavior (see below).

In Figure 2, we consider two representative example for which we compare the quadrupole (blue lines) and octupole (red lines) levels of approximation, where we consider the time evolution of the eccentricity and inclination. In both of these examples, we consider a $1 M_{\odot}$ star orbited by an eccentric Jupiter, with a test particle on a far-away orbit. One can consider such a setting to represent a result of a scattering event, for example.

On the left set of panels of Figure 2, we consider a Jupiter at 0.4 au with $e_{1}=0.65$ and a test particle at 7 au with $e_{2}=0.4$, initialized on a retrograde orbit $\left(i=91^{\circ}\right)$. With the introduction of the octupole level of approximation to the calculation, the test particle eccentricity starts to oscillate, though in this case it never increases pass its initial value (due to choice of initial conditions here). More notably, the test particle inclination, with respect to the total angular momentum, oscillates from retrograde $\left(>90^{\circ}\right.$ which was the initial condition) to prograde $\left(<90^{\circ}\right)$. As in the more general case, there is no apparent associated timescale for this flipping modulation, and it seems chaotic in nature (see below). While the quadrupole-level is circulatory in nature (see $i-\Omega_{2}$ plot), a libration behavior emerges at the octupole level.

On the right set of panels of Figure 2, we consider a Jupiter at 3 au with $e_{1}=0.85$ and a test particle at 50 au with $e_{2}=0.7$, and the system is initialized on a prograde orbit $\left(i=20^{\circ}\right)$. Here, like the previous example, the outer test particle eccentricity begins to oscillate and even grows above the initial value. However, unlike the previous example, this system does not flip. The inclination does oscillate with a longscale modulation, and we show the long-scale evolution that captures about four octupole cycles. The system does not exhibit a chaotic behavior in this case, and it remains in a circulatory trajectory even after the inclusion of the octupole level of approximation to the calculation.

In Figure 3, we zoom-in on the evolution of a different example and also provide the time evolution of $\omega_{2}$ for the octupole level itself. As in the general hierarchical secular three body problem, we find the short-scale (associated with the quadrupole) oscillations that are modulated by the higher-level octupole approximation. The octupole modulations take place on timescales that are between a few $\times t_{\text {quad }}$ to a few tens $\times t_{\text {quad }}$.

It is interesting to note that the inclination flips shown in Figures 2 and 3 are qualitatively different from the ordered, back-and-forth oscillation of the quadrupole-level of approximate evolution. The latter produces a simple, ordered oscillation of the inclination angle between $i_{0}$ and $180^{\circ}-i_{0}$, 


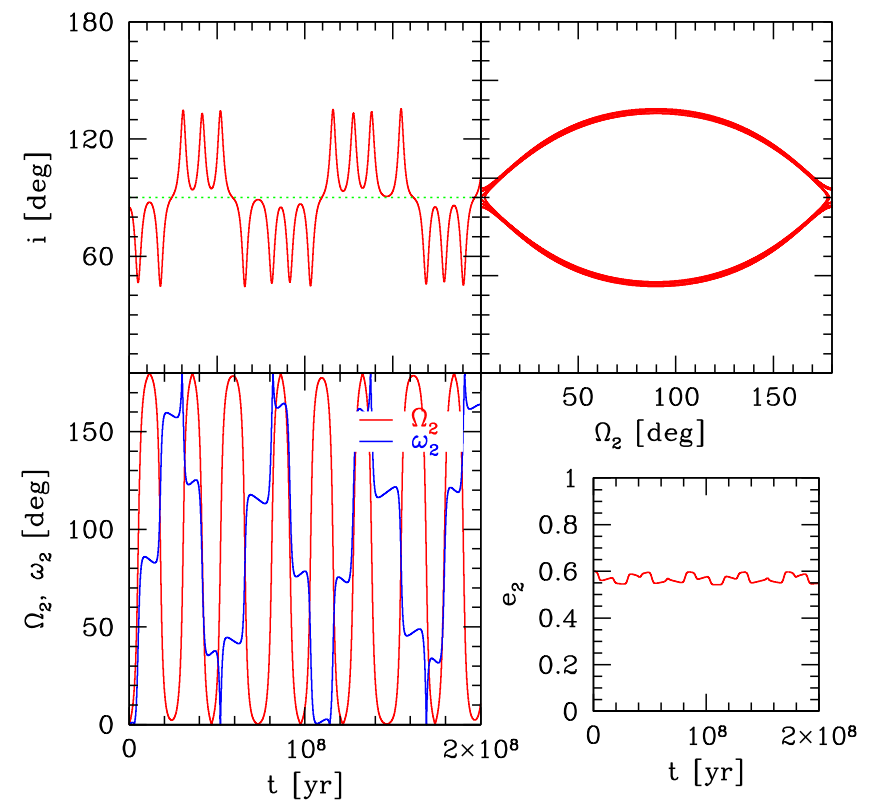

Figure 3. We consider the system: $m_{1}=1 M_{\odot}, m_{2}=1 M_{j}, a_{1}=0.5 \mathrm{au}, a_{2}=$ $10 \mathrm{au}, e_{1}=0.4$ and $e_{2}=0.6$. We initialize the system with $\omega_{2}=\Omega_{2}=0^{\circ}$ and $i=85^{\circ}$. We show the time evolution of the orbital parameters, i.e., argument of pericenter $\omega_{2}$, longitude of ascending node $\Omega_{2}$, inclination $i$ and the outer orbital eccentricity, $e_{2}$. We also consider the inclination evolution as a function of $\Omega_{2}$. Here, both $\Omega_{2}$ and $\omega_{2}$ were folded to achieve the $0^{\circ}-180^{\circ}$ symmetry.

for the librating regime. However, in the presence of the octupole level of approximation, the system behaves similarly to the general flips discussed in $\mathrm{NaOz}$ et al. (2011), where the inclination oscillates for sometime at the prograde $\left(i<90^{\circ}\right)$ regime and then flips to the retrograde configuration $\left(i>90^{\circ}\right)$.

The eccentricity, $e_{2}$, gives rise to an additional complication, as the outer test particle eccentricity can essentially grow so much that the orbits will cross. We adopt the nominal stability criterion

$$
\epsilon=\frac{a_{1}}{a_{2}} \frac{e_{2}}{1-e_{2}^{2}}<0.1
$$

to guide us when the system leaves stability. We discuss this stability criterion in the context of $\mathrm{N}$-body comparisons in Appendix B.

To explore the chaotic nature of the system and the different dynamical regimes, we use surface of sections. The outer test particle approximation reduces the general hierarchical three body system from six degrees of freedom to four degrees of freedom. In addition, in the test particle limit, the inner orbit is stationary and reduces the system to two degrees of freedom. In this system, $f$ and $\delta$ are the only conserved parameters, and $\omega_{2}$ and $\Omega_{2}$ are the only coordinates that can change with time. For a two-degrees-of-freedom system, the surface of section projects a four-dimensional trajectory on a two-dimensional surface, where we select intersections of the trajectories on the surfaces when $\omega_{2}$ and $\Omega_{2}$ move in the positive directions. For simplicity, we separate the two initial conditions into three characteristic parameters: $e_{1}$, the inner orbit eccentricity (which remains constant during the evolution), the energy (or initial value of the reduced Hamiltonian $f$ ), and

$$
\delta=\frac{m_{1}-m_{2}}{m_{1}+m_{2}} \frac{a_{1}}{a_{2}} e_{1}
$$

Note that the energy depends on $\delta$ and $e_{1}$, so in fact, although we choose to characterize the surface of sections by three parameters, there are only two independent ones.

In Figures 4-6, we consider the surface of sections for various values of the $f, e_{1}$, and $\delta$ in the $J_{2}-\omega_{2}$ plane (top row in each figure) and in the $J_{2, z}-\Omega_{2}$ plane (bottom row in each figure). In both planes, we identify the resonances at which the momenta and angles undergo bound oscillations. The trajectories in this region are quasi-periodic, and the system is in the libration mode. The circulation region represents trajectories where the angles are not constrained between two specific values. Both librating and circulatory trajectories are mapped onto a one-dimensional manifold on the surface of section, and they form lines on the section. However, chaotic trajectories are mapped onto a higher dimensional manifold and they are filling an area on the surface. We note that some of the trajectories in the figures, due to sampling limitation, seem to appear as dashed lines, but they actually represent a one-dimensional manifold. In all of the maps, we indicate the instability regime (light orange stripe) for which $\epsilon>0.1$.

We intersect the trajectories at $\Omega_{2}=\pi / 2$ to produce the surfaces in the $J_{2}-\omega_{2}$ plane, in order to capture the librating cases. The empty regions at large $J_{2}$ at the parameter spaces in the far left and right panels in Figure 5 (i.e., for the parameters: $e=0.4, \delta=0.02$ and $f= \pm 10$ ) and the far left panel in Figure 6 (for the parameters: $e=0.9, \delta=0.1$ and $f=-10$ ) correspond to regions with no physical solutions. The variabilities in $J_{2}$ are mostly small in the stable regime. We see that there is regular behavior, i.e., trajectories that fill onedimensional lines on the surface of section in most of the stable regions. We find the emergence of chaos in parts of the unstable zones, in particular when $J_{2}$ is low ( $e_{2}$ is high).

Considering the $J_{2, z}-\Omega_{2}$ plane, we intersect the trajectories at $\omega_{2}=0$. The system exhibits a chaotic behavior across the entire parameter regime of $e_{1}, \delta$, and $f$. Most of the circulation region, associated with curved, non chaotic one-dimensional manifold, are typically associated with $\left|J_{2, z}\right| \gtrsim 0.3$. The outer orbits can flip ( $J_{2, z}$ shifts signs) in most of the parameter space.

Resonances can be easily identified in a few of the maps. Specifically, in the $J_{2}-\omega_{2}$ plots, the resonances can be found centered near $\boldsymbol{\omega}_{2}=\pi$ (e.g., $e_{1}=0.4, \delta=0.02, f=-10$, and $e_{1}=0.9, \quad \delta=0.1, \quad f=-10$, etc.), and $\omega_{2}=\pi / 2 \quad$ (e.g., $e_{1}=0.4, \delta=0.02, f=10$ ). The dynamics is quite complicated when $e_{1}$ is higher and when $\delta$ is larger, and higher-order resonances (appearing as small liberating islands) emerge in the surface of section in the $J_{2}-\omega_{2}$ plane when $e_{1}=0.9, \delta=0.1$, and $f=0$. Resonances can also be identified in the $J_{2, z}-\Omega_{2}$ plane, such as $e_{1}=0.9, \delta=0.1, f=10$.

\section{The Role of the GR}

As noted previously in many studies, GR precession tends to suppress the inner orbit eccentricity excitations associated with the Eccentric Kozai-Lidov (EKL) mechanism, and thus suppress the flips (e.g., Naoz et al. 2013b). In our secular case, the inner orbit is massive and the outer orbit is a test particle, so practically the inner orbit does not feel the outer orbit gravitational interactions. However, the inner orbit can still precess due to GR with the nominal precession rate (e.g., 

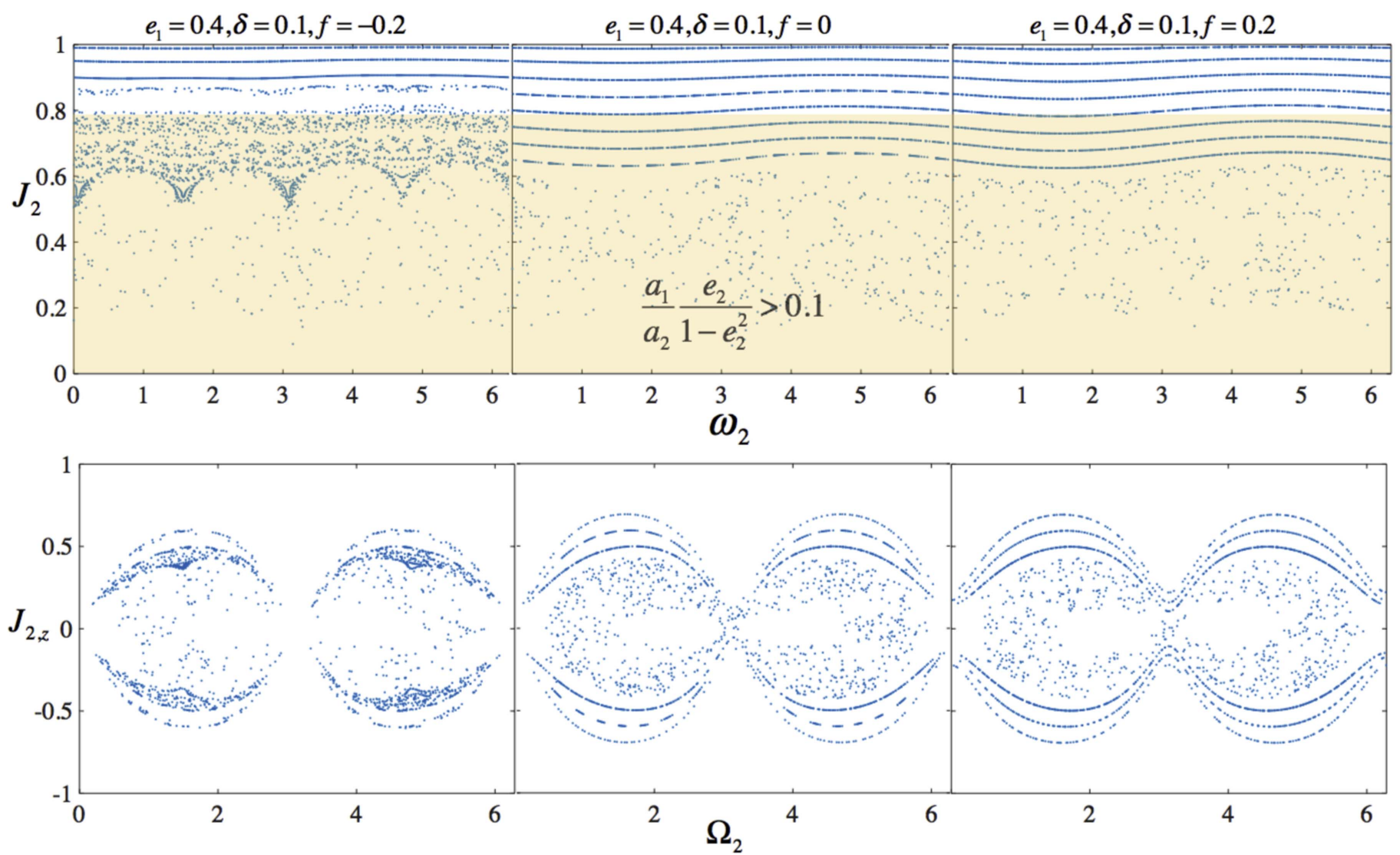

Figure 4. Surface of section. We consider $e_{1}=0.4, \delta=0.1$ and, from left to right $f=-0.2,0$ and 0.2 . We note that cases $f=10$ and $f=-10$ give a similar map to the $f=0.2$ and $f=-0.2$ cases, respectively, and thus were not depicted, to avoid clutter. The light orange stripe marks the unstable regime, for which $\epsilon>0.1$.

Naoz et al. 2013b)

$$
\left.\frac{d \omega_{1}}{d t}\right|_{\mathrm{GR}, \text { inner }}=\frac{3 k^{3}\left(m_{1}+m_{2}\right)^{3 / 2}}{a_{1}^{5 / 2} c^{2}\left(1-e_{1}^{2}\right)}
$$

where $k^{2}$ is the gravitational constant and $c$ is the speed of light. However, in our frame of reference, where the inner orbit carries all of the angular momentum, we are basically working in the rotating frame of the inner orbit. Therefore, since we set $\omega_{1}=-\pi-\Omega_{2}$, (see Appendix A), GR precession of $\omega_{1}$ translates to a precession of $\Omega_{2}$. Thus, using our coordinate transformation we find

$$
\left.\frac{d \Omega_{2}}{d t}\right|_{\mathrm{GR}, \Omega_{2}}=-\frac{3 k^{3}\left(m_{1}+m_{2}\right)^{3 / 2}}{a_{1}^{5 / 2} c^{2}\left(1-e_{1}^{2}\right)}
$$

which can suppress the inclination oscillations. The timescale associated with that precession is the nominal GR one

$$
t_{\mathrm{GR}, \Omega_{2}} \sim 2 \pi \frac{a_{1}^{5 / 2} c^{2}\left(1-e_{1}^{2}\right)}{3 k^{3}\left(m_{1}+m_{2}\right)^{3 / 2}} .
$$

In Figure 7 , we consider two examples, one for which $t_{\mathrm{GR}, \Omega_{2}} \sim 5 \times 10^{6}$ year is smaller than $t_{\text {quad }} \sim 6 \times 10^{6}$ year (top panel), and the other of which $t_{\mathrm{GR}, \Omega_{2}} \sim 4.4 \times 10^{8}$ year is a bit longer than the corresponding $t_{\text {quad }} \sim 2 \times 10^{7}$ year (bottom panel). Both of these examples had a Sun-sized star and a Jupiter-sized planet, orbited by a far-away test particle. The Jupiter has a non-negligible eccentricity that perhaps can be a result of either a scattering event or a high-eccentricity migration. In the top panel, the Jupiter was set at $a_{1}=0.5 \mathrm{au}$ with $e_{1}=0.4$ and the test particle was set at $a_{2}=10$ au and $e_{2}=0.6$. The system, in the absence of GR, was in libration mode and, as noted before, exhibited a chaotic nature. However, the GR precession bound the system into a circulatory regime and suppressed the flips. In the bottom panel, the Jupiter was set at $a_{1}=3$ au and $e_{1}=0.9$, while the test particle was set at $a_{2}=40$ au with $e_{2}=0.65$. Note that in this latter case, although the Jupiter is rather far from the host star and the GR precession timescale is longer than the secular precession timescale, the GR changes the dynamics. Specifically, before the inclusion of GR precession, the system was in a libration mode and seemed quasi-periodic. However, after the inclusion of GR, the system exhibits both libration and circulation, and the emergence of chaotic behavior seems to take place. The dramatic change in dynamical behavior with the inclusion of GR precession, even if it takes place on longer timescales than the secular timescales, was noted previously in both the general and inner test particle cases in Naoz et al. (2013b).

We note that in all of our calculations below, we also take into account the outer orbit GR precession (e.g., Naoz et al. 2013b)

$$
\left.\frac{d \omega_{2}}{d t}\right|_{\mathrm{GR}, \text { outer }}=\frac{3 k^{3}\left(m_{1}+m_{2}\right)^{3 / 2}}{a_{2}^{5 / 2} c^{2}\left(1-e_{2}^{2}\right)},
$$

which typically takes place on much larger timescales. 

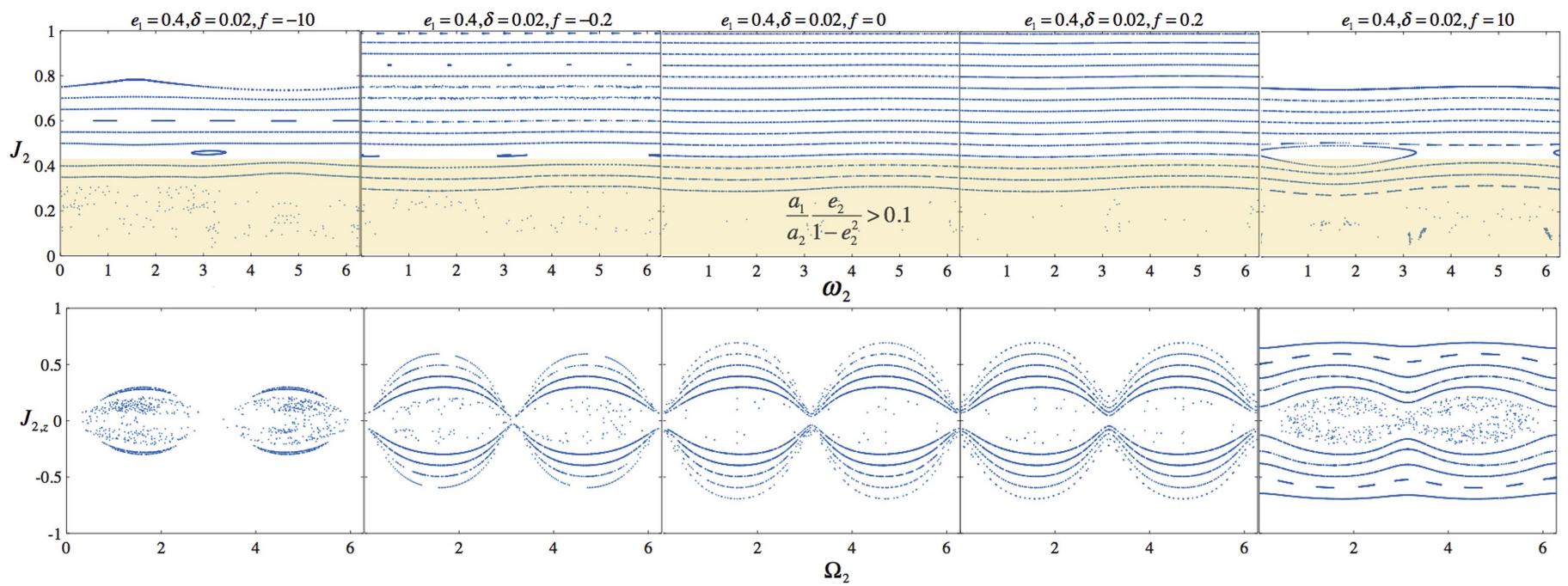

Figure 5. Surface of section. We consider $e_{1}=0.4, \delta=0.02$ (compared to Figure 4, this means changing the factor $\left(m_{1}-m_{2}\right) a_{1} / a_{2} /\left(m_{1}+m_{2}\right)$ by factor of 5) and, from left to right $f=-10,-0.2,0,0.2$, and 10. The light orange stripe marks the unstable regime, for which $\epsilon>0.1$.

\section{A Study Case Application: Individual Debris Disk Particles}

Debris disks mark the late end stages of planet formation and are made of the leftover material of rocks and ices. The gravitational interactions between these particles and interior or exterior companions can leave a distinct imprint on the morphology of the disk and can cause dust production (e.g., Matthews et al. 2014; Rodigas et al. 2014; Nesvold \& Kuchner 2015; Lee \& Chiang 2016; Nesvold et al. 2016; Nesvold et al. 2017). Many of these studies typically focus on a few million years of integration to allow for comparison of observations, which usually can detect young systems. Here, we allow for longer integration timescales and investigate the evolution of a test particle under the influence of an eccentric Jupiter.

In Figure 8, we show an example system, where we consider an eccentric Jupiter at 5 au, with 0.85 eccentricity orbiting a one-solar-mass star. The test particle is located at 55 au and initialized with an eccentricity of 0.5 . We integrate the octupole level equations of motion in the presence of GR precession for both the inner and outer orbits. As in the example depicted in Figure 7, which also considered icy-body or comet reservoirs analogs, the orbit switches between libration and circulation, as can be seen in the bottom panel.

We consider the effect of the planet's eccentricity, $e_{1}$, and the test particle inclination by surveying the parameter space of $e_{1}$ and initial inclination for a given system, where we set $a_{2}=55 \mathrm{au}$, with $e_{2}=0.5$, and set the system initially with $\Omega_{2}=90^{\circ}$ and $\omega_{2}=0^{\circ}$. Many giant exoplanets have high eccentricities, specifically for giant planets $\left(m \sin i>0.1 M_{J}\right.$, with separation $>0.05 \mathrm{au}$ ) the average eccentricity is $\sim 0.2$, and a maximum value of $0.97 .^{6}$ Thus, an eccentric Jupiter doesn't seem like an unlikely configuration for a planetary system. In the example depicted in Figure 8, the time evolution of the test particle's inclination that starts with a moderate eccentricity oscillates between extreme values $20^{\circ} \sim 160^{\circ}$ without increasing its eccentricity.

The system depicted in Figure 8 is, of course, just one example for a particular choice of the orbital parameters. To

6 Taken from The Exoplanet Orbit Database (Wright et al. 2011). study the effects of planet's eccentricity and initial inclination, we have systematically explored the $e_{1}-i$ parameter space in Figure 9. We choose a Jupiter-like system $\left(a_{1}=5 \mathrm{au}\right)$ and for a range of eccentricities, with an outer orbit at $55 \mathrm{au}$. The test particle orbit was initialized with $e_{2}=0.5, \Omega_{2}=90^{\circ}, \omega_{2}=0^{\circ}$, and a range of inclinations. We show the maximum inclination reached during the evolution as a function of the initial inclination and eccentricity in Figure 9. In the left panel, we depict the initial inclination versus the initial Jupiter's eccentricity, where the color code marks the maximum inclination reached. The solid line in the left panel follows Equation (19), which is consistent in that the resonance associated with the quadrupole-level of approximation is indeed the main driver for the dynamical evolution of the system. In the figure, we depict the initial inclination regime to the prograde case $\left(i_{\text {initial }} \leqslant 90^{\circ}\right)$ to avoid clutter. However, in the right panel, we show the maximum inclination reached during the evolution as a function of the initial inclination going all the way to $180^{\circ}$ this time.

As a proof of concept, we depict in Figure 10 the behavior of a narrow debris disk after 4 Gyr of evolution. This inclination represents the instantaneous inclination at $4 \mathrm{Gyr}$ of evolution. The system, of course, continues to oscillate, and the disk of particles will remain puffed. The inclination and eccentricity of the system at this snapshot are qualitatively different from the initial conditions assumed. This hypothetical system shows the orbital configuration of a disk located between 55 and $65 \mathrm{au}$, with an interior eccentric Jupiter $\left(e_{1}=0.85\right)$ at $5 \mathrm{au}$ around a solar-mass star. The system was set initially with a mutual inclination of $20^{\circ}, e_{2}=0.3$ and $\Omega_{2}$ and $\omega_{2}$ are chosen from a random uniform distribution between $0^{\circ}-360^{\circ}$. While the particles in the disk have eccentric values, the disk does not appear as a coherent eccentric ring, as the values of $\Omega_{2}$ and $\omega_{2}$ are random. At the end of the integration, the particles in the disk became slightly more eccentric (with an average eccentricity of $\sim 0.34$ ), and there is a clear trend of $\Omega_{2}$ as a function of inclination. The particles with inclination above the initial $20^{\circ}$ have a value of $\Omega_{2}$ close to zero (or with the symmetric value $180^{\circ}$ ), while the particles with inclination below $20^{\circ}$ have $\Omega_{2}$ values closer to $90^{\circ}$. The behavior is singular to $\Omega_{2}$ and has not manifested itself in $\omega_{2}$ (it was not 

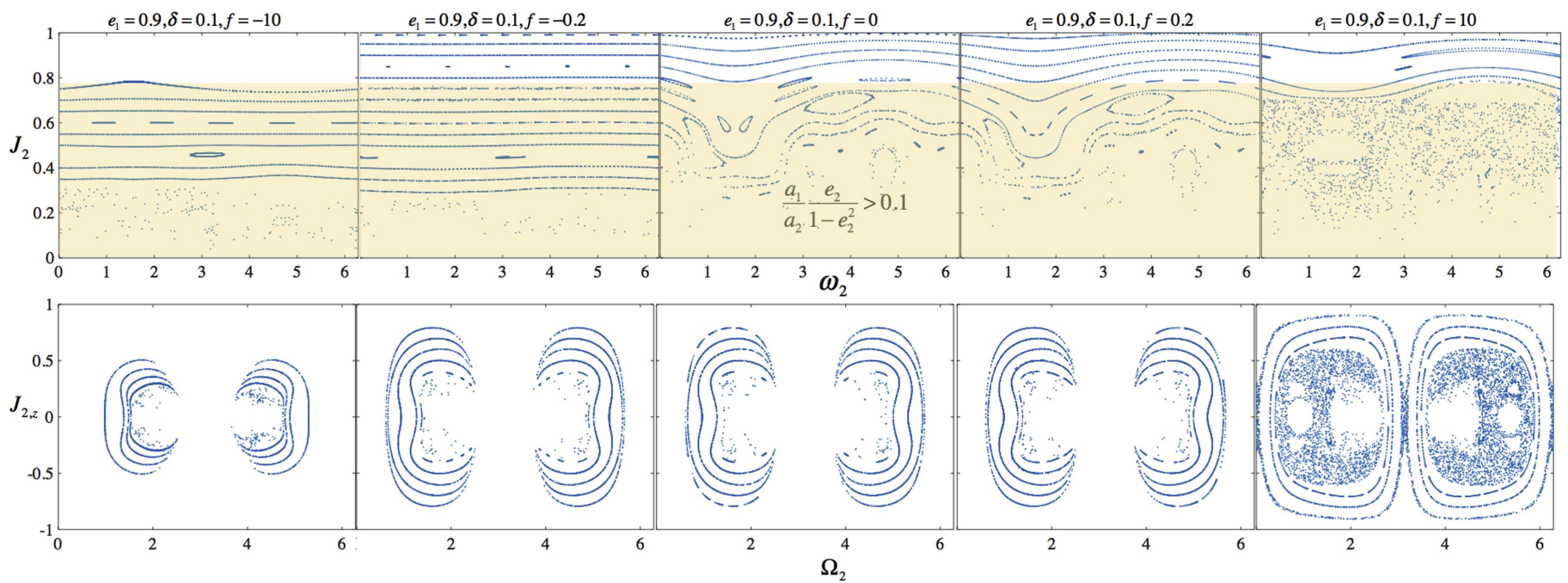

Figure 6. Surface of section. We consider $e_{1}=0.9, \delta=0.1$ and, from left to right $f=-10,-0.2,0,0.2$, and 10 . The light orange stripe marks the unstable regime, for which $\epsilon>0.1$
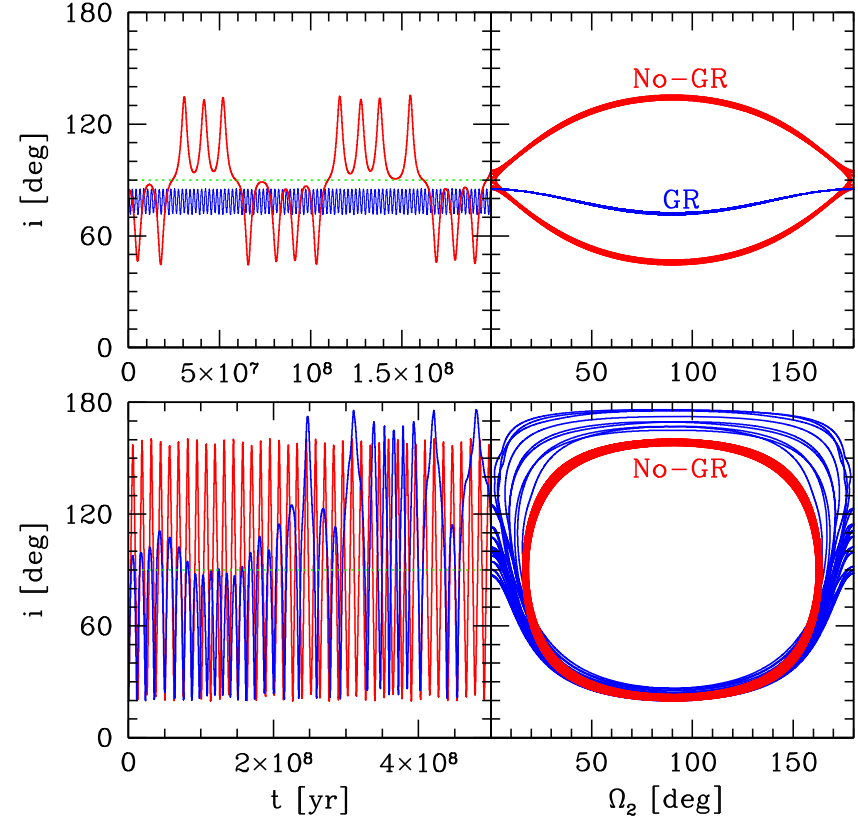

Figure 7. The role of GR. We consider two cases, the evolution without GR (red lines) and the evolution with GR (blue lines). Top panel: we consider the following system: $m_{1}=1 M_{\odot}, m_{2}=1 \mathrm{M}_{j}, a_{1}=0.5 \mathrm{au}, a_{2}=10 \mathrm{au}, e_{1}=0.4$ and $e_{2}=0.6$. We initialize the system with $\omega_{2}=\Omega_{2}=0^{\circ}$ and $i=85^{\circ}$. For this system, we find that $t_{\mathrm{GR} \text {,inner }} \sim 5 \times 10^{6}$ year, which is much shorter than the quadrupole timescale. Bottom panel: we consider the following system: $m_{1}=1 M_{\odot}, m_{2}=1 M_{j}, a_{1}=3 \mathrm{au}, a_{2}=40 \mathrm{au}, e_{1}=0.9$ and $e_{2}=0.65$. We initialize the system with $\omega_{2}=90^{\circ}$ and $\Omega_{2}=100^{\circ}$ and $i=20^{\circ}$. The GR precession timescale for this system is estimated as $t_{\mathrm{GR}, \text { inner }} \sim 4.4 \times 10^{8}$ year, which is longer than the quadrupole timescale. This is a typical situation to an individual debris disk particle or an icy-body reservoir object (see Section 6).

depicted here to avoid clutter). We note that some retrograde particles were formed as well, in line with the Zanardi et al. (2017) numerical results of an orbital flip. These behaviors are easily understandable from the surface of section maps depicted above.

Its important to note that during the evolution of the system depicted in Figure 10, the maximum $\epsilon$ achieved was 0.0625 . The average value of $\epsilon$, which corresponds to the maximum $e_{2}$ achieved during the evolution, was $\sim 0.4$. Thus, the system is

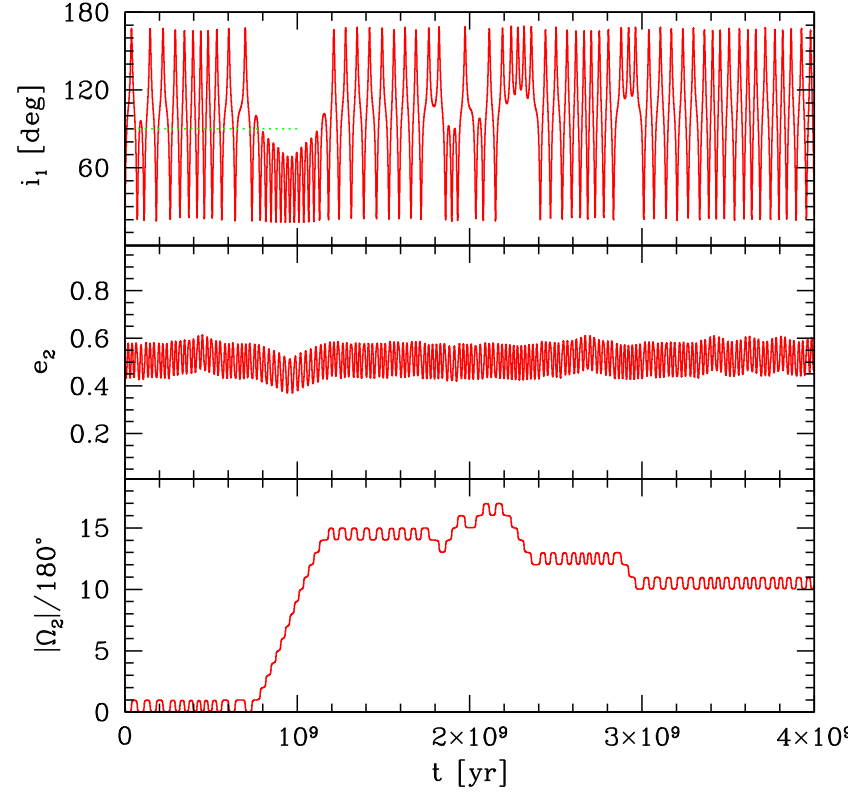

Figure 8. Debris disk particles. We consider the time evolution of a test particle located at 55 au from a $M_{\odot}$ star due to the gravitational perturbations from an eccentric Jupiter $a_{1}=5$ au and $e_{1}=0.85$. We initialized the system with $e_{2}=0.5, \Omega_{2}=90^{\circ}, \omega_{2}=0^{\circ}$, and $i=20^{\circ}$. We consider from top to bottom the inclination, eccentricity, and $\Omega_{2}$. The transition between librating and circulating can clearly be seen in the bottom panel. When the angle is in circulation mode, it increases in value as a function of time.

kept stable during this evolution, the secular approximation holds, and we do not expect any scattering event. We also note that we have compared a debris disk particles secular and $\mathrm{N}$-body evolution and found a qualitative agreement, which is similar in behavior to the left panel of Figure 11, and this is not shown here to avoid clutter.

\section{Discussion}

We have studied the secular evolution of an outer test particle hierarchical system. We presented the three body, outer test body Hamiltonian up to the octupole level of approximation in the power series of the semimajor axis ratio. We showed that in the quadrupole-level of approximation, 

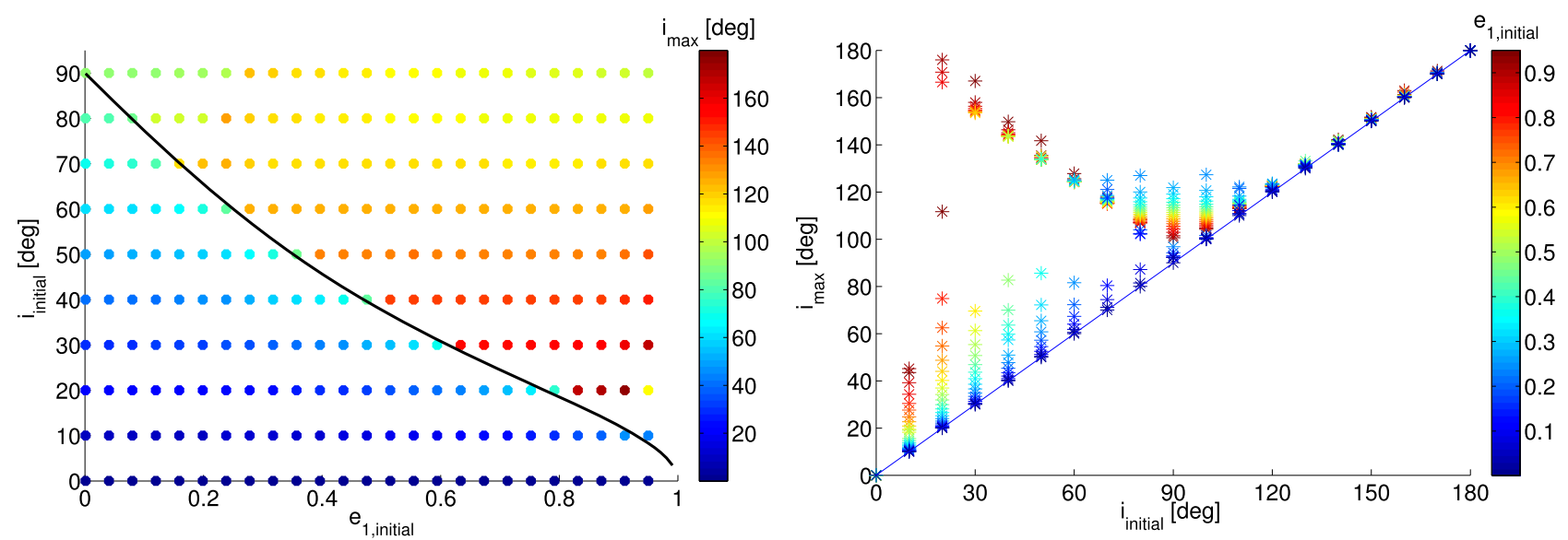

Figure 9. Debris disk particles. We consider a Sun-Jupiter-like system, setting Jupiter at 5 au. We set the test particle at 55 au, with $e_{2}=0.5$. We systematically vary the inclination and Jupiter's eccentricity. We initially set the system with $\Omega_{2}=90^{\circ}$ and $\omega_{2}=0^{\circ}$. The left panel shows the initial condition map (inclination vs. the Jupiter's eccentricity) where the color code depicts the maximum inclination the system reached during its evolution of 4 Gyr. The solid line shows the analytical equation to reach $90^{\circ}$, Equation (19). The right panel shows the maximum inclination reached as a function of the initial inclination (that goes all the way to $180^{\circ}$ ). The color code here marks the inner orbit's eccentricity.

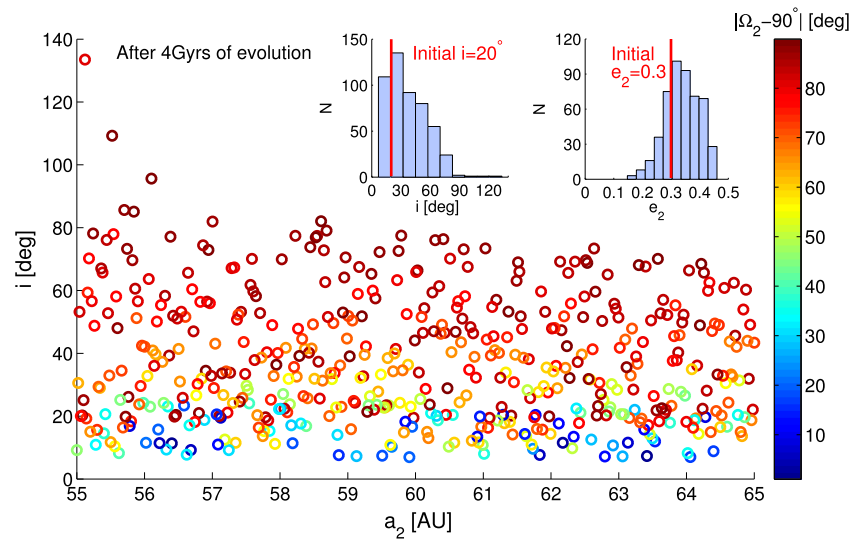

Figure 10. Long timescale evolution of a debris disk. The mutual inclination, $i$ as a function of the semimajor axis, $a_{2}$, after $4 \mathrm{Gyr}$ of integration. The color code shows the longitude of ascending nodes $\left|\Omega_{2}-90^{\circ}\right|$ at that time (this presentation emphasizes the symmetry in the system). The right inset shows the histogram of the final eccentricity of the disk, while the left inset shows the histogram of the final mutual inclination of the disk. As a proof of concept, we consider a narrow debris disk located between 55 and 65 au, with an interior eccentric Jupiter $\left(e_{1}=0.85\right)$ at 5 au around a solar-mass star. The system was initially set with a mutual inclination of $20^{\circ}, e_{2}=0.3$ and $\Omega_{2}$ and $\omega_{2}$ are chosen from a random uniform distribution between $0^{\circ}-360^{\circ}$. The results depicted here were achieved by integrating over the equations of motion, Equations (11)-(14). Note that GR effects are included here as well.

$\left(a_{1} / a_{2}\right)^{2}$, the system has two distinct behaviors, librating and circulating (see Figure 1), where the librating mode gives the nominal precession of the nodes, results for which the inclination oscillates between the $i_{90}$ inclination (the inclination for which $\Omega_{2}=90^{\circ}$ and $180^{\circ}-i_{90}$ ). Furthermore, the bound values of the liberating mode have a simple analytical expression, Equation (21). We also found the minimum and maximum inclination that the system can reach in the circulating mode (see Equations (19) and (20)). These conditions are sensitive to the initial inner orbit's eccentricity and are nicely reproduced in numerical testing here (see Figure 9) and in Zanardi et al. (2017) numerical experiments (see their Figure 14). We also estimated the timescale for oscillations for the two modes (see Section 3.2).

We then showed that introducing the octupole level of approximation allows for transition between the two libration and circulation modes (see Figures 2 and 3). This yields that the overall dynamics of the system is similar to the behavior of the general flip behavior in the EKL mechanism. In particular, the dynamics is quite chaotic for parameter regions with high $e_{2}$ and perpendicular mutual inclinations (when $J_{2}$ is low and when $J_{2, z}$ is near zero), as shown in the surface of sections Figures 4-6.

GR can play an important role in suppressing or exciting the eccentricities in the hierarchical three body problem (e.g., Naoz et al. 2013b). We find here similar behavior. Specifically, the inclination excitation will be suppressed for systems with GR precession faster than the quadrupole precession. However, when GR precession takes place on similar (or even somewhat larger) timescales to that of the quadrupole precession, the additional precession can produce inclination excitations, in a non-regular manner (see Figure 7).

The dynamics of these type of systems can have a wide range of applications, from stars around supermassive black hole binaries to the evolution of individual debris disk particles. We have chosen the latter as an example and presented a typical example of the evolution of a test particle due to the gravitational perturbations from an eccentric Jupiter (see Figure 8). We systematically varied the Jupiter's eccentricity and the outer orbit's inclination, where we found an agreement between the analytical relation for crossing the $90^{\circ}$ threshold and the numerical tests. This also suggests that an eccentric planet can pump up the inclination of icy-body or cometreservoir analogs (Figure 9). This was further supported by considering the evolution of an initially narrow, thin disk of test particles exterior to an eccentric planet, with initial mutual inclination of $20^{\circ}$. The disk became puffed with some particles on a retrograde orbits (see Figure 10). A detailed study of the effects of eccentric planets on exterior test particle is presented in Zanardi et al. (2017). This mechanism will also be important, for example, to circumbinary planetary systems (considered first by Ziglin 1975) and the stars near mergers of black hole binaries. However, more detailed studies are required that are beyond the scope of this paper.

We thank the referee for a quick and detailed report, and especially for his/her inquiry about the surface of sections. We also thank Vladislav Sidorenko for pointing out some missing 

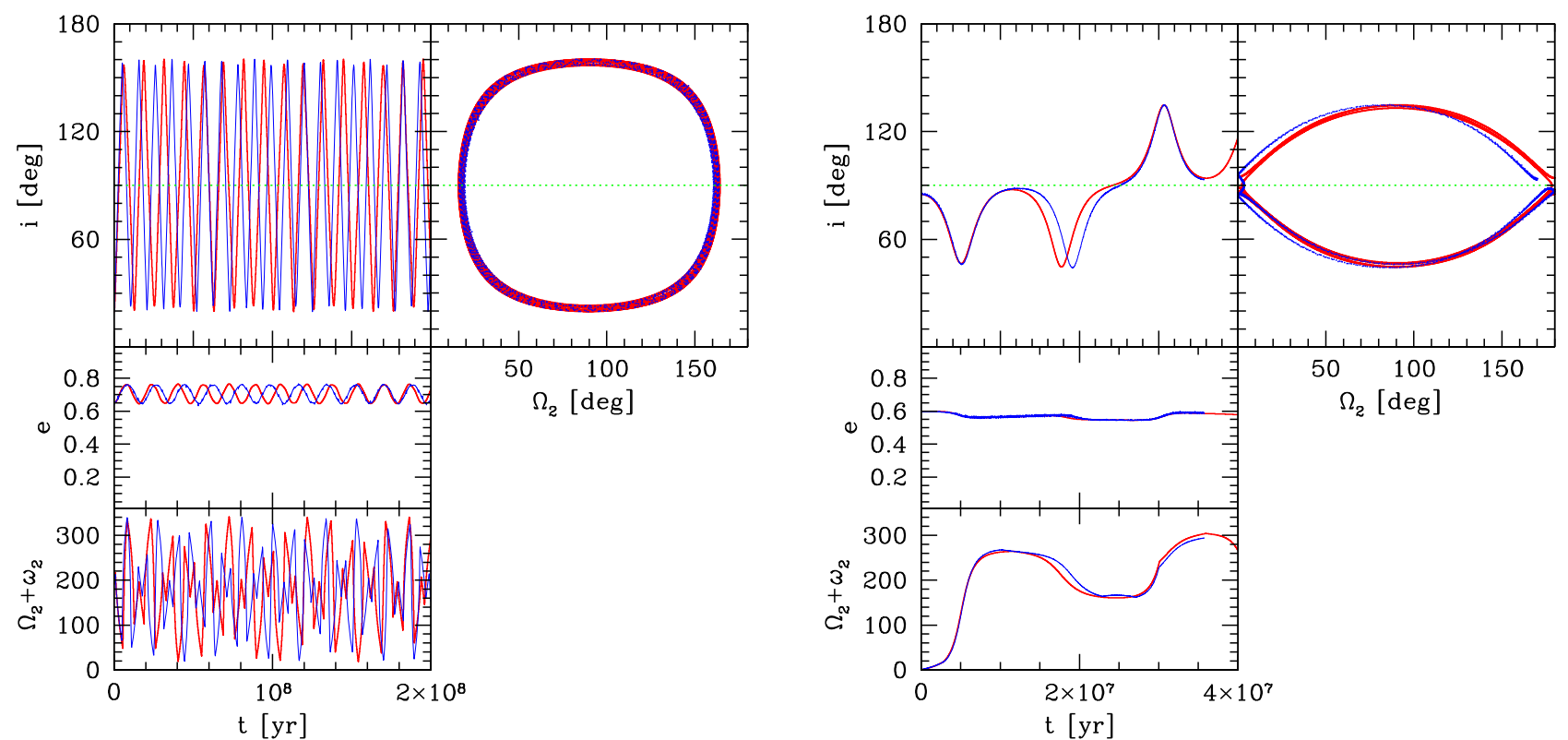

Figure 11. Comparison between the $N$-body results and the secular approximation at the octupole level. We show the inclination, eccentricity, and $\Omega_{2}+\omega_{2}$ as a function of time in the left-hand side and $i-\Omega_{2}$ in the right-hand panel. Red lines correspond to the secular calculation (up to the octupole level of of approximation) and blue lines corresponds to the $N$-body calculation. Note that in the $i-\Omega_{2}$, we depict the $N$-body results as points to allow for an easier comparison. On the left side, we consider the following system: $m_{1}=1 M_{\odot} m_{2}=1 M_{J}, a_{1}=0.3$ au, $a_{2}=40$ au, $e_{1}=0.9, e_{2}=0.65, \omega_{2}=90^{\circ}, \Omega_{2}=100^{\circ}$, and $i=20^{\circ}$. On the right side, we consider the following system: $m_{1}=1 M_{\odot} m_{2}=1 M_{J}, a_{1}=0.5 \mathrm{au}, a_{2}=10 \mathrm{au}, e_{1}=0.4, e_{2}=0.6, \omega_{2}=\Omega_{2}=0^{\circ}$, and $i=85^{\circ}$. The evolution of this system was depicted in Figure 3, and here we show this system for a shorter evolution timescale, to allow for a better comparison between the $N$-body and secular calculation.

references. S.N. acknowledges partial support from a Sloan Foundation Fellowship. G.L. is supported in part by the Harvard William F. Milton Award. M.Z., G.dE., and R.P.D. acknowledge the financial support given by IALP, CONICET, and Agencia de Promoción Científica, through the PIP 0436/ 13 and PICT 2014-1292.

\section{Appendix A \\ Orbital Parameters and the Scaled Time}

One might have expected that $\omega_{1}=$ const when the outer particle is massless. But in truth, $\Omega_{1}$ is undefined because the reference plane is aligned with the inner orbit. Therefore, the inner planet must only have $\omega_{1}+\Omega_{1}=$ const, and we may choose without loss of generality the constant to equal zero. Hence, elimination of the nodes (i.e., $\Omega_{1}-\Omega_{2}=\pi$ ) implies $\omega_{1}=-\pi-\Omega_{2}$.

Similarly to the treatment done in Lithwick \& Naoz (2011), we have rescaled the momenta by an arbitrary constant to achieve the specific angular momentum. The Hamiltonian is rescaled by the same constant, and we find that the rescaled time is:

$$
\begin{aligned}
\tau & =\frac{t}{16} \frac{m_{1} m_{2}}{\left(m_{1}+m_{2}\right)^{2}} \sqrt{\frac{G_{N}\left(m_{1}+m_{2}\right)}{a_{2}^{3}}}\left(\frac{a_{1}}{a_{2}}\right)^{2} \\
& =\frac{t}{16} \frac{m_{1} m_{2}}{\left(m_{1}+m_{2}\right)^{2}} \frac{2 \pi}{P_{2}}\left(\frac{a_{1}}{a_{2}}\right)^{2},
\end{aligned}
$$

where $t$ is the true time. The numerical factor 16 comes by taking $m_{3} \rightarrow 0$ in the general Hamiltonian (see Naoz 2016, for the general form of the hierarchical three body double averaged Hamiltonian). There is a choice to be made, to either scale the Hamiltonian by this numerical factor or $\tau$. Here, we choose to absorb this number in $\tau$ to be consistent with the inner test particle Hamiltonian.
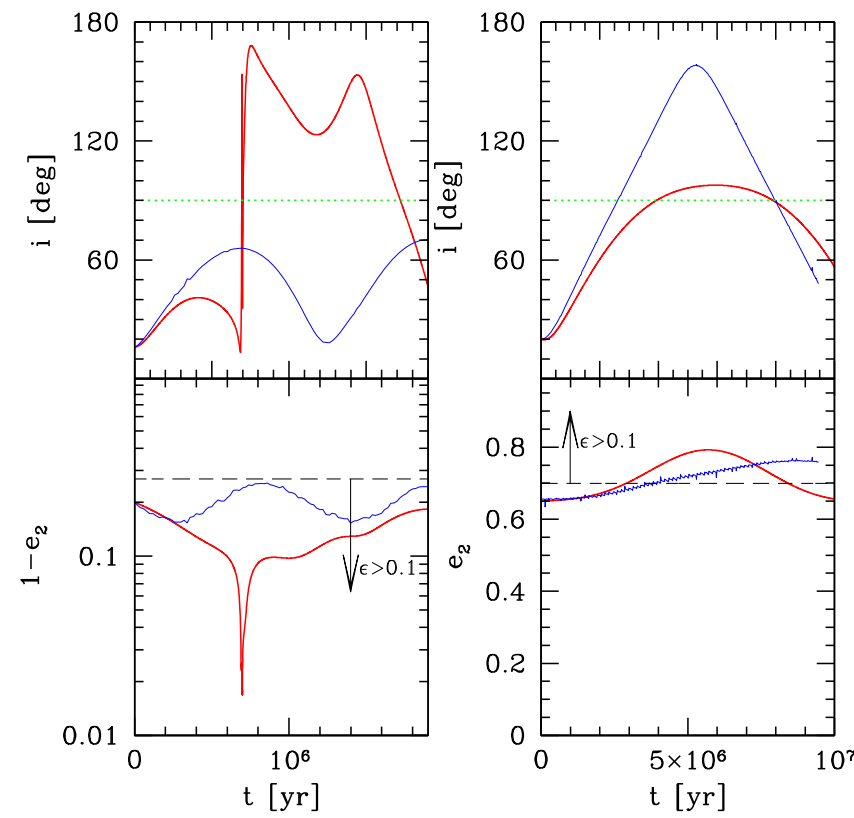

Figure 12. Comparison between the $N$-body results and the secular approximation at the octupole level for systems around $\epsilon \sim 0.1$. We show the inclination and eccentricity. Red lines correspond to the secular calculation (up to the octupole level of of approximation) and blue lines corresponds to the $N$-body calculation. Left side: we consider the following system: $m_{1}=1 M_{\odot} m_{2}=1 M_{J}, a_{1}=1 \mathrm{au}$, $a_{2}=15 \mathrm{au}, e_{1}=0.8, e_{2}=0.8, \omega_{2}=0^{\circ}, \Omega_{2}=90^{\circ}$, and $i=16^{\circ}$. These parameters imply an initial $\epsilon$ of 0.148 . Right side: we consider the following system: $m_{1}=1 M_{\odot} m_{2}=1 M_{J}, a_{1}=3 \mathrm{au}, a_{2}=40 \mathrm{au}, e_{1}=0.9, e_{2}=0.65$, $\omega_{2}=90^{\circ}, \Omega_{2}=100^{\circ}$, and $i=20^{\circ}$. The latter systems initialize with $\epsilon=0.084$; however, as time goes by the eccentricity grows and the approximation breaks.

\section{Appendix B \\ Comparison with $N$-body}

In this section, we compare the secular approximation at the octupole level in the test particle limit with the $N$-body 
simulation, using Mercury code (Chambers \& Migliorini 1997). In this comparison, we did not include GR. Good agreements can be reached when the apocenter distance of the inner binary is much smaller than the pericenter distance of the outer binary. In particular, we include an illustrative example here in Figure 11, where we consider an eccentric $(e=0.9$ Jupiter at $3 \mathrm{au}$ around a solar-like object). The test particle is set at $40 \mathrm{au}$ with $e_{2}=0.65$. The system is set initially with $i=20^{\circ}$, $\omega_{2}=90^{\circ}$ and $\Omega_{2}=100^{\circ}$.

As shown in Figure 11, the secular approximation (shown as red lines) agrees qualitatively well with the $N$-body results for the eccentricity and inclination oscillations. This is likely due to the double averaging process, but nonetheless, the maximum and minimum of the orbital parameters are conserved in both $\mathrm{N}$-body and secular calculations. It is interesting to note that similarly to (Lithwick \& Naoz 2011), the approximation holds as long as $\epsilon<0.1$. However, unlike the inner test particle case, $e_{2}$ can change and increase during the evolution which may break the validity of the approximation during the evolution. This is shown in Figure 12.

\section{References}

Blaes, O., Lee, M. H., \& Socrates, A. 2002, ApJ, 578, 775

Chambers, J. E., \& Migliorini, F. 1997, BAAS, 29, 1024

de la Fuente Marcos, C., de la Fuente Marcos, R., \& Aarseth, S. J. 2015, MNRAS, 446, 1867

Farago, F., \& Laskar, J. 2010, MNRAS, 401, 1189

Ford, E. B., Kozinsky, B., \& Rasio, F. A. 2000, ApJ, 535, 385
Gallardo, T. 2006, Icar, 181, 205

Gallardo, T., Hugo, G., \& Pais, P. 2012, Icar, 220, 392

Harrington, R. S. 1968, AJ, 73, 190

Harrington, R. S. 1969, CeMec, 1, 200

Innanen, K. A., Zheng, J. Q., Mikkola, S., \& Valtonen, M. J. 1997, AJ, 113, 1915

Katz, B., Dong, S., \& Malhotra, R. 2011, PhRvL, 107, 181101

Kozai, Y. 1962, AJ, 67, 591

Lee, E. J., \& Chiang, E. I. 2016, ApJ, 827, 125

Li, D., Zhou, J.-L., \& Zhang, H. 2014a, MNRAS, 437, 3832

Li, G., Naoz, S., Holman, M., \& Loeb, A. 2014b, arXiv:1405.0494

Li, G., Naoz, S., Kocsis, B., \& Loeb, A. 2014c, ApJ, 785, 116

Lidov, M. L. 1962, P\&SS, 9, 719

Lithwick, Y., \& Naoz, S. 2011, ApJ, 742, 94

Matthews, B. C., Krivov, A. V., Wyatt, M. C., Bryden, G., \& Eiroa, C. 2014, in Protostars and Planets VI, ed. H. Beuther et al. (Tucson, AZ: Univ. Arizona Press), 521

Murray, C. D., \& Dermott, S. F. 2000, Solar System Dynamics (Cambridge: Cambridge Univ. Press)

Naoz, S. 2016, ARA\&A, 54, 441

Naoz, S., Farr, W. M., Lithwick, Y., Rasio, F. A., \& Teyssandier, J. 2011, Natur, 473, 187

Naoz, S., Farr, W. M., Lithwick, Y., Rasio, F. A., \& Teyssandier, J. 2013a, MNRAS, 431, 2155

Naoz, S., Kocsis, B., Loeb, A., \& Yunes, N. 2013b, ApJ, 773, 187

Nesvold, E. R., \& Kuchner, M. J. 2015, ApJ, 798, 83

Nesvold, E. R., Naoz, S., \& Fitzgerald, M. P. 2017, ApJL, 837, L6

Nesvold, E. R., Naoz, S., Vican, L., \& Farr, W. M. 2016, ApJ, 826, 19

Rodigas, T. J., Malhotra, R., \& Hinz, P. M. 2014, ApJ, 780, 65

Teyssandier, J., Naoz, S., Lizarraga, I., \& Rasio, F. A. 2013, ApJ, 779, 166 Verrier, P. E., \& Evans, N. W. 2009, MNRAS, 394, 1721

Wright, J. T., Fakhouri, O., Marcy, G. W., et al. 2011, PASP, 123, 412

Zanardi, M., de Elía, G. C., Di Sisto, R. P., et al. 2017, arXiv:1701.03865

Ziglin, S. L. 1975, SvAL, 1, 194 